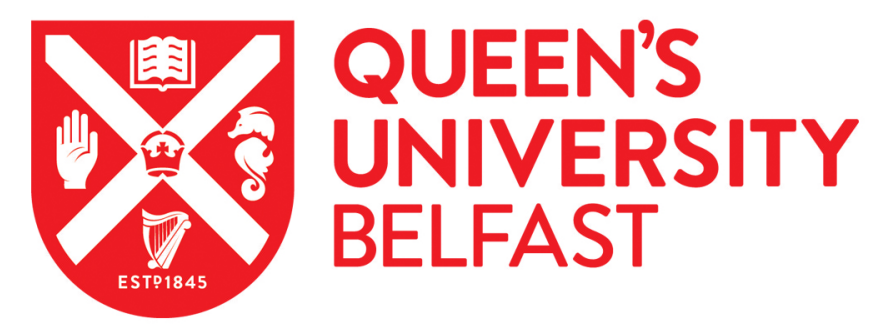

\title{
Electrostatic shock structures in dissipative multi-ion dusty plasmas
}

Elkamash, I. S., \& Kourakis, I. (2018). Electrostatic shock structures in dissipative multi-ion dusty plasmas. Physics of Plasmas, 25(6), 1-11. [062104]. https://doi.org/10.1063/1.5029322

Published in:
Physics of Plasmas

Document Version:

Peer reviewed version

Queen's University Belfast - Research Portal:

Link to publication record in Queen's University Belfast Research Portal

Publisher rights

Copyright 2018 AIP Publishing. This work is made available online in accordance with the publisher's policies. Please refer to any applicable terms of use of the publisher.

\section{General rights}

Copyright for the publications made accessible via the Queen's University Belfast Research Portal is retained by the author(s) and / or other copyright owners and it is a condition of accessing these publications that users recognise and abide by the legal requirements associated with these rights.

Take down policy

The Research Portal is Queen's institutional repository that provides access to Queen's research output. Every effort has been made to ensure that content in the Research Portal does not infringe any person's rights, or applicable UK laws. If you discover content in the Research Portal that you believe breaches copyright or violates any law, please contact openaccess@qub.ac.uk. 


\title{
Electrostatic shock structures in dissipative multi-ion dusty plasmas
}

\author{
I. S. Elkamash ${ }^{1,2}$ and I. Kourakis ${ }^{1}$
}

1 Centre for Plasma Physics, Queen's University Belfast, BT7 1 NN Northern Ireland, UK
2 Physics Department, Faculty of Science, Mansoura University, 35516 Mansoura, Egypt

(Dated: May 29, 2018)

\begin{abstract}
A comprehensive analytical model is introduced for shock excitations in dusty bi-ion plasma mixtures, taking into account collisionality and kinematic (fluid) viscosity. A multicomponent plasma configuration is considered, consisting of positive ions, negative ions, electrons and a massive charged component in the background (dust). The ionic dynamical scale is focused upon, thus electrons are assumed to be thermalized, while the dust is stationary. A dissipative hybrid Korteweg de Vries/Burgers (hKdV-B) equation is derived. An analytical solution is obtained, in the form of a shock structure (a step-shaped function for the electrostatic potential, or an electric field pulse) whose maximum amplitude in the far downstream region decays in time. The effect of relevant plasma configuration parameters, in addition to dissipation, is investigated. Our work extends earlier studies of ion-acoustic type shock waves in pure (two-component) bi-ion plasma mixtures.
\end{abstract}

\section{INTRODUCTION}

Multicomponent plasma configurations, containing electrons, positive and negative ions, in addition to nanometer or even micron-sized charged particulates (dust "grains"), are widely used in microelectronics, in optoelectronics, in photonics, in microelectromechanical devices, in material synthesis and in other industrial and technological processes, e.g. in manufacturing miniature circuit chips and various other applications [1-3]. By using a sophisticated experimental approach, Takeuchi et al [4] have shown that a negative ion plasma can be produced by introducing a small amount of SF6 gas into a collisionless plasma with $K^{+}$ions and electrons. They found that positive (compression) and negative (rarefaction) density jumps are generated by applying a positive and negative potential ramp, while the steepening of the initial density jump depends on the actual negative ion concentration in the plasma. Luo et al [5] subsequently showed that if the presence of concentration of negative ions in plasma is higher than $90 \%$, the pulse perturbation tends to steepen and compressive electrostatic shocks are formed. In the presence of negative charge dust grains, compressive electrostatic shock waves have been observed in a double plasma device [6]. Also, they have showed that the phase velocity of the electrostatic shock wave increases with an increase in the dust density. Bandyopadhyay et al [7] observed long-lived dust acoustic (DA) waves in a fluid-state dusty plasma and showed that the velocity of DA wavepackets increases, while their width decreases, for higher values of the driving modulating voltage. Sarma and Nakamura [8] showed experimentally that the concentration of the negative ions in dusty plasma may affect the polarity of electrostatic shock waves. They predicted that both compressive and rarefactive electrostatic shock wave may exist, for a given initial excitation at a critical concentration of the negative ions. Heinrich et al [9] later used high-speed video imaging to study self-excited dust acoustic shock waves observed in a dc glow discharge. Recently, by using a supersonic flow of charged microparticles, a finite amplitude dust acoustic shock wave was observed in a strongly coupled laboratory dusty plasma [10]. The balancing between wave steepening due to an increase in the dust density and dissipation due to dust-neutral collisions and viscosity effects was thus shown experimentally to lead to the formation of steady state shockwaves.

Collective effects in the form of linear and nonlinear modes in dusty plasmas, have been the focus of a large body of theoretical and experimental research in the last two decades, mainly [11-15]. A Korteweg - de Vries/Burgers (KdV-B) type equation was derived by Shukla [16] by adding a kinematic viscosity force to the fluid equation of motion, to model electrostatic shocks and holes in dusty plasma analytically. As regards dusty plasma containing negative ions, which is our primary focus here, the linear instability of ion acoustic waves has been investigated via a comprehensive hydrodynamic model by Vladimirov et al [17]. Rosenberg and Merlino [18] investigated the influence of charged dust grains on the stability of ion acoustic waves in a dusty negativeion plasma, discussing both laboratory and space plasma environments. The effect of ion-dust collisions and ion kinematic viscosity on the formation of DIA solitary and shock waves in a dusty plasma in presence of negative ions has been studied in Ref. 19 .

The purpose of this paper is to investigate the occurrence and to study the propagation characteristics of electrostatic shock wave in negative-ion plasma in the presence of dust particles. In Sec. II we present a plasmahydrodynamic model for our plasma configuration of interest. A linear analysis is presented in Sec. III. Using a perturbation method, a new evolution equation is derived for the electrostatic potential perturbation in Sec. IV. In Sec. V, an investigation of the role of various plasma plasma parameters is presented. A summary and critical discussion of our findings is provided in Sec. VI. 


\section{THE MODEL}

We consider two distinct ion fluids evolving against a neutralizing background consisting of thermal (Boltzmann distributed) electrons and dust grains. The plasma components interact with each other through mutual ionion collisions, ion-dust collisions and a viscous drag due to interparticle collisions and the collective electrostatic potential. Since the electron thermal speed is much larger than that of either of the ion species and the (ionic) dynamical velocity scale of interest is slow, we have neglected the electron inertia, viz. $n_{e}=n_{e 0} \exp \left(e \phi / k_{B} T_{e}\right)$, (where $\mathrm{e}$ is the magnitude of the electron charge, $\phi$ the wave potential, $k_{B}$ is the Boltzmann constant and $T_{e}$ the electron temperature). The dust thermal speed is much lower than the wave's phase velocity and its response to electrostatic perturbations is slow, hence we consider the dust component as a stationary background. Furthermore, we assume that the dust particles have a constant mass $m_{d}$ and the dust grain charge is assumed to be fixed $z_{d}\left(q_{d}=\right.$ const $)$, since the charging rate is negligibly low, compared to the dynamical plasma response rate $\left(\nu_{c h} \gg \omega_{p}\right)$ [11]. An adiabatic thermal pressure term is considered, as we have assumed that the ion thermal velocity is less than the characteristic phase velocity and the electron thermal speed.

Adopting a one dimensional (1D) planar geometry, for simplicity, the fluid model equations read:

$$
\begin{aligned}
\frac{\partial n_{1}}{\partial t}+\frac{\partial}{\partial x}\left(n_{1} u_{1}\right) & =0 \\
m_{1} n_{1}\left(\frac{\partial u_{1}}{\partial t}+u_{1} \frac{\partial u_{1}}{\partial x}\right) & =-z_{1} e n_{1} \frac{\partial \phi}{\partial x}-\frac{\partial P_{1}}{\partial x} \\
-m_{1} n_{1} \nu_{12}\left(u_{1}-u_{2}\right) & +m_{1} n_{1} \eta_{1} \frac{\partial^{2} u_{1}}{\partial x^{2}}-\nu_{1 d} u_{1}, \\
\frac{\partial n_{2}}{\partial t}+\frac{\partial}{\partial x}\left(n_{2} u_{2}\right) & =0, \\
m_{2} n_{2}\left(\frac{\partial u_{2}}{\partial t}+u_{2} \frac{\partial u_{2}}{\partial x}\right) & =z_{2} e n_{2} \frac{\partial \phi}{\partial x}-\frac{\partial P_{2}}{\partial x} \\
-m_{2} n_{2} \nu_{21}\left(u_{2}-u_{1}\right) & +m_{2} n_{2} \eta_{2} \frac{\partial^{2} u_{2}}{\partial x^{2}}-\nu_{2 d} u_{2}, \\
\frac{\partial^{2} \phi}{\partial x^{2}}=-\frac{1}{\epsilon_{0}} e\left(z_{1} n_{1}\right. & \left.-z_{2} n_{2}-n_{e}+s_{d} z_{d} n_{d}\right)
\end{aligned}
$$

where the first two (continuity and momentum) equations describe the positive ion fluid, the following two sister equations describe the negative ion fluid and the final one (Poisson's equation) describes the electrostatic potential evolution under the influence of charge variations in space and time $\left(\epsilon_{0}\right.$ denotes the susceptibility of vacuum, as usual). The physical quantities $n_{j}, u_{j}$ respectively denote the number density and the fluid speed, of fluid(s) $j=1,2$ (i.e., the positive ion fluid and the negative ion fluid ), while $m_{j}$ and $z_{j}$ obviously denote the respective mass and charge state, in the usual way. Note that we have adopted no prior assumption for the dust charge sign, which may be either positive or negative, for $s_{d}=q_{d} /\left|q_{d}\right|= \pm 1$, respectively. We have defined the characteristic coefficients $\nu_{j}$ (denoting the inter-ionfluid collisional frequency), $\nu_{j d}$ (the ion-dust collision frequency) and $\eta_{j}$, representing the intrinsic plasma kinematic viscosity. Finally, the ion thermal effects are included in the model through the thermal pressure variables, denoted by $P_{1}$ and $P_{2}$ for the respective ion fluids. The system of equations $(6-10)$ is closed by assuming an explicit density dependence of the pressure term(s) in the form $p_{j}=C n_{j}^{\gamma}$, where $\gamma$ is the ratio of specific heats. Combining this assumption with the equation of state (at equilibrium) $p_{j, 0}=n_{j 0} k_{B} T_{j}$ (where $T_{j}$ denotes the temperature of species $j$ ), the pressure term in the momentum Eqs. (7) and (9) is rearranged as $\nabla p_{j} / n_{j}=\gamma K_{B} T_{j} n_{j 0}^{1-\gamma} n_{j}^{\gamma-2} \nabla n_{j}$ with the adiabatic index $\gamma=(2+f) / f$ (where $f$ denotes the number of degrees of freedom), i.e. $\gamma=3$ in our case.

The model equations may be cast in a dimensionless form, for simplicity in algebraic manipulation. Adopting appropriate scales, the normalized evolution equations become:

$$
\begin{aligned}
\frac{\partial n_{1}}{\partial t}+\frac{\partial}{\partial x}\left(n_{1} u_{1}\right) & =0 \\
\frac{\partial u_{1}}{\partial t}+u_{1} \frac{\partial u_{1}}{\partial x} & =-\frac{\partial \phi}{\partial x}-\sigma_{1} n_{1} \frac{\partial n_{1}}{\partial x} \\
& -\nu_{12}\left(u_{1}-u_{2}\right)+\eta_{1} \frac{\partial^{2} u_{1}}{\partial x^{2}}-\nu_{1 d} u_{1} \\
\frac{\partial n_{2}}{\partial t}+\frac{\partial}{\partial x}\left(n_{2} u_{2}\right) & =0 \\
\frac{\partial u_{2}}{\partial t}+u_{2} \frac{\partial u_{2}}{\partial x} & =\mu \frac{\partial \phi}{\partial x}-\sigma_{2} n_{2} \frac{\partial n_{2}}{\partial x} \\
& -\nu_{21}\left(u_{2}-u_{1}\right)+\eta_{2} \frac{\partial^{2} u_{2}}{\partial x^{2}}-\nu_{2 d} u_{2} \\
\frac{\partial^{2} \phi}{\partial x^{2}} & =\delta_{e} n_{e}-n_{1}+\delta_{i} n_{2}-s_{d} \delta_{d}
\end{aligned}
$$

The rescaled electron density reads $n_{e}(\phi)=e^{\phi} \approx 1+\phi+$ $\phi^{2} / 2+\phi^{3} / 6+\ldots$ We have defined the quantities:

$$
\mu=\frac{q_{2} / m_{2}}{q_{1} / m_{1}}, \quad \text { and } \quad \sigma_{j}=\frac{3}{z_{1}} \frac{T_{j}}{T_{e}} \frac{m_{1}}{m_{j}},
$$

i.e.,

$$
\sigma_{1}=\frac{3}{z_{1}} \theta_{1}, \quad \text { and } \quad \sigma_{2}=\sigma_{1} \frac{T_{2}}{T_{1}} \frac{m_{1}}{m_{2}} .
$$

where $q_{1}=z_{1} e, q_{2}=z_{2} e, \theta_{1}=\frac{T_{1}}{T_{e}}, e$ is the elementary charge unit and $z_{1}, z_{2}$ are the charge state of positive and negative ions, respectively. Time $t$ and space $x$ have been normalized by (the positive ion plasma frequency) $\omega_{p, 1}=\left(z_{1}^{2} e^{2} n_{1,0} / \epsilon_{0} m_{1}\right)^{1 / 2}$ and (the positive ion Debye length) $\lambda_{D, 1}=\left(\epsilon_{0} k_{B} T_{e} / z_{1} e^{2} n_{1,0}\right)^{1 / 2}$. The number density $n_{j}$ and fluid speed $u_{j}$ variables are normalized by the respective unperturbed number density $n_{j 0}$ (for each fluid; viz. $j=e, 1,2$ for electrons, ions 1 and ions 2) and by the characteristic speed $c_{s}=\left(z_{1} K_{B} T_{e} / m_{1}\right)^{1 / 2}$ (i.e., essentially the sound speed in $e-i$ plasmas). The quantities $\phi, \eta_{j}$ and $\nu_{j}$ are normalized by $k_{B} T_{e} / e, \lambda_{D 1}^{2} \omega_{p, 1}$ and $\omega_{p, 1}$, respectively. We have defined the relative ion number density ratio $\delta_{i}=\frac{z_{2} n_{20}}{z_{1} n_{10}}$, the electron-to-(positive)ion number density ratio $\delta_{e}=\frac{n_{e 0}}{z_{1} n_{10}}$ and the dust den- 
sity ratio $\delta_{d}=\frac{z_{d} n_{d}}{z_{1} n_{10}}$. Neutrality at equilibrium (where $\left.n_{j, 0}=1, \quad \forall j\right)$ imposes the condition

$$
\delta_{e}=1-\delta_{i}+s_{d} \delta_{d} .
$$

Finally, we have assumed the ordering $\nu_{12} \sim \epsilon^{3 / 2}, \nu_{j d} \sim$ $\epsilon^{3 / 2}$ and $\eta_{j} \sim \epsilon^{1 / 2}(j=1,2)$, suggesting that both ion-ion and dust-ion collisional frequencies and the viscosity coefficient are very low, compared to intrinsic plasma quantities (i.e. the plasma frequency, for the former quantities).

\section{LINEAR WAVE ANALYSIS}

Linearizing the dimensionless system of evolution equations (6) - (10) and Fourier transforming, we readily obtain the dielectric function

$$
D(\omega, k)=1+\frac{\delta_{e}}{k^{2}}-\frac{1}{\omega^{2}-\sigma_{1} k^{2}}-\frac{\delta_{i} \mu}{\omega^{2}-\sigma_{2} k^{2}},
$$

where we clearly distinguish the electron, positive ion and negative ion contributions in the second, third and fourth term(s) in the right-hand side (rhs), respectively. The normal modes are obtained by setting $D(\omega, k)=0$. One is thus led to the dispersion relation

$$
\omega^{4}+\Gamma_{1} \omega^{2}+\Gamma_{0}=0
$$

where $\Gamma_{0}=\frac{k^{4}}{k^{2}+\delta_{e}}\left[\delta_{i} \mu \sigma_{1}+\sigma_{2}+\sigma_{1} \sigma_{2}\left(k^{2}+\delta_{e}\right)\right]$ and $\Gamma_{1}=-\frac{k^{2}}{k^{2}+\delta_{e}}\left[1+\delta_{i} \mu+k^{2}\left(\sigma_{1}+\sigma_{2}\right)+\left(\sigma_{1}+\sigma_{2}\right) \delta_{e}\right]$. Two positive expressions are thus obtained from the latter relation for the frequency (square), in the form: $\omega_{ \pm}^{2}=\frac{1}{2}\left(-\Gamma_{1} \pm \sqrt{\Gamma_{1}^{2}-4 \Gamma_{0}}\right)$. It is straightforward to find that $\omega_{ \pm} \rightarrow 0$ for both modes: these essentially represent a fast and a slow ion-acoustic mode, as expected $[20,21]$, here actually modified in the presence of the dust. In the absence of the negative ions $\delta_{i}=0$, one recovers the dust-ion acoustic wave dispersion relation:

$$
\omega^{2}=\frac{k^{2}}{k^{2}+1+s_{d} \delta_{d}}+\sigma_{1} k^{2},
$$

as expected [11].

Based on the expression(s) of the dispersion relation Eq.14, we have analysed the effect of the negative ion density on the linear dispersion relation. As shown in Fig. 1, the dispersion relation (14) provides two acoustic modes (and no Langmuir-like mode, as intuitively expected). In the presence the negative ions $\delta_{i} \neq 0$, the slow and fast mode respectively lie below and above the plain ion-acoustic frequency, i.e. $\omega_{p,-}<\left.\omega\right|_{\delta_{i}=0}<\omega_{p,+}$. The effect of the dust density is studied in Fig. 2. It is clearly visible in Fig. 2a that the presence of positively charged dust $\left(s_{d}=1\right)$ leads to a decrease in the frequency of both linear modes. On the other hand, introducing negatively charged dust particles into the plasma $\left(s_{d}=-1\right)$ increases the frequency of both modes, as shown in Fig. $2 \mathrm{~b}$.

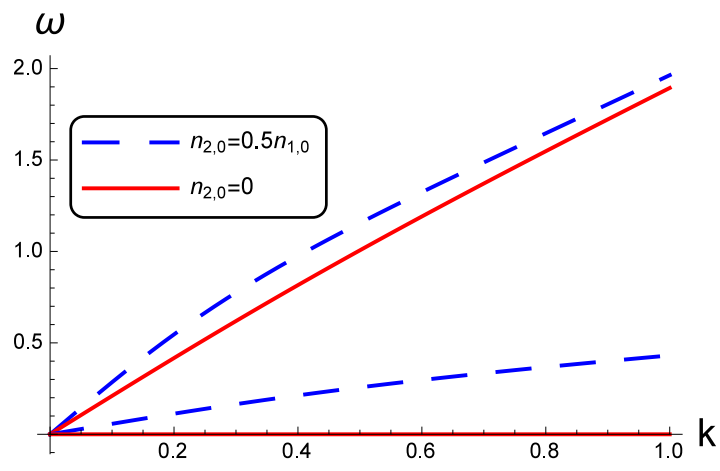

FIG. 1: (Color online) Dispersion relation: the effect of the negative ion concentration $\left(n_{2,0}\right)$ is depicted, for $s_{d}=-1$, $n_{d}=1.7 \times 10^{6} \mathrm{~cm}^{-3}$ and $n_{1,0}=2 \times 10^{9} \mathrm{~cm}^{-3}$. The other parameters are $z_{1}=z_{2}=1, z_{d}=350, m_{1}=39 m_{p}, m_{2}=$ $146 m_{p}$ ( $m_{p}$ : proton mass), $T_{e}=T_{1}=0.2 \mathrm{eV}$ and $T_{2}=T_{e} / 8$, [22]. The dimensionless parameters are $\delta_{d}=0.3, \delta_{i}=0.5$, $\mu=0.267, \sigma_{1}=3$ and $\sigma_{2}=0.1$.

\section{DERIVATION OF KDVB EQUATION}

Anticipating small amplitude nonlinear ion acoustic waves, we have adopted the reductive perturbation technique [23] by introducing the stretched coordinates:

$$
\xi=\epsilon^{\frac{1}{2}}(x-V t), \quad \tau=\epsilon^{\frac{3}{2}} t .
$$

Here, $V$ is a real (free) parameter, denoting the phase velocity of ion acoustic waves (to be determined later by algebraic compatibility considerations) and $\epsilon(\epsilon \ll 1)$ is a small real expansion parameter $(0<\epsilon \ll 1)$ characterizing the strength of the nonlinearity. We expand the perturbed state variables appearing in Eqs. (6-10) in terms of the smallness parameter $\epsilon$ as follows

$$
\begin{aligned}
n_{j} & =1+\epsilon n_{j}^{(1)}+\epsilon^{2} n_{j}^{(2)}+\epsilon^{3} n_{j}^{(3)}+\ldots, \\
u_{j} & =\epsilon u_{j}^{(1)}+\epsilon^{2} u_{j}^{(2)}+\epsilon^{3} u_{j}^{(3)}+\ldots \\
\phi & =\epsilon \phi^{(1)}+\epsilon^{2} \phi^{(2)}+\epsilon^{3} \phi^{(3)}+\ldots,
\end{aligned}
$$

(for $j=1,2$ ). We proceed by inserting the expansion(s) Eqs. (17) into Eqs. (6)-(10)), and then isolating different contributions arising in different orders (powers of $\epsilon$ ).

To lowest order, we have obtained precidely Eqs. (A1)(A5); see in Appendix A. Upon integrating, and applying appropriate boundary conditions, i.e., assuming that $n_{1,2}^{(1)}=1$ and $u_{1,2}^{(1)}=\phi^{(1)} \rightarrow 0$ for $x \rightarrow \pm \infty$, we obtain the algebraic relations

$$
\begin{aligned}
n_{1}^{(1)} & =\frac{1}{V^{2}-\sigma_{1}} \phi^{(1)}, \\
n_{2}^{(1)} & =-\frac{\mu}{V^{2}-\sigma_{2}} \phi^{(1)}, \\
u_{1}^{(1)} & =\frac{V}{V^{2}-\sigma_{1}} \phi^{(1)} \\
\text { and } \quad u_{2}^{(1)} & =-\frac{\mu V}{V^{2}-\sigma_{2}} \phi^{(1)} .
\end{aligned}
$$

Combining the expressions Eqs. (18) and (19) with Eq. (A5), we obtain an expression for the phase velocity of 


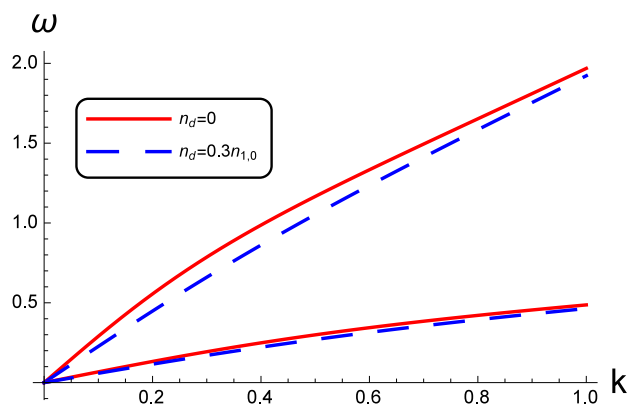

(a)

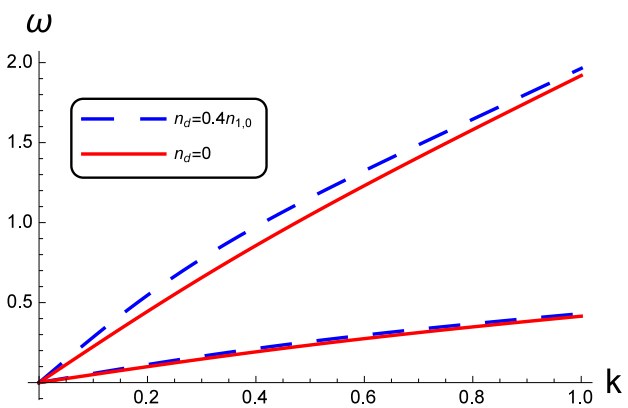

(b)

FIG. 2: (Color online) Dispersion relation: the effect of (a) the positive dust density $\left(s_{d}=1\right)$, and (b) the negative dust density $\left(s_{d}=-1\right)$ is depicted. The parameter values adopted are: $n_{2,0}=1.6 \times 10^{9} \mathrm{~cm}^{-3}$ and $n_{1,0}=2 \times 10^{9} \mathrm{~cm}^{-3}, z_{1}=z_{2}=1, z_{d}=350$, $m_{1}=39 m_{p}, m_{2}=146 m_{p}$ ( $m_{p}$ : proton mass), $T_{e}=T_{1}=0.2 \mathrm{eV}$ and $T_{2}=T_{e} / 8$. The dimensionless parameters are $\delta_{d}=0.3$, $\delta_{i}=0.5, \mu=0.267, \sigma_{1}=3$ and $\sigma_{2}=0.1$.

the ion acoustic shocks in the form:

$$
V^{4}+a_{1} V^{2}+a_{0}=0
$$

where

$$
\begin{aligned}
& a_{1}=-\frac{1+\delta_{i} \mu+\delta_{e}\left(\sigma_{1}+\sigma_{2}\right)}{\delta_{e}}, \\
& a_{0}=\frac{\delta_{i} \mu \sigma_{1}+\sigma_{2}+\delta_{e} \sigma_{1} \sigma_{2}}{\delta_{e}} .
\end{aligned}
$$

Remember that $\delta_{e}$ is prescribed by Eq. (12). The wave phase speed is thus given by the expressions

$$
V_{ \pm}^{2}=\frac{1}{2}\left(-a_{1} \pm \sqrt{a_{1}^{2}-4 a_{0}}\right)
$$

in agreement with the considerations for $\omega_{ \pm}$. As a mat- ter of fact, it is straightforward to verify that $V_{ \pm}=$ $\lim _{k \rightarrow 0}\left(\omega_{ \pm} / k\right)$; indeed, careful inspection reveals that (25) may be precisely obtained from (14) upon formally setting $\omega \rightarrow k V$ and then taking the limit $k \rightarrow 0$.

From next order in $\epsilon$, along with Eq. 25, we obtain after a tedious algebraic procedure (the lengthy details can be found in the Appendix) the following equation

$$
\frac{\partial \psi}{\partial \tau}+A \psi \frac{\partial \psi}{\partial \xi}+B \frac{\partial^{3} \psi}{\partial \xi^{3}}=C \frac{\partial^{2} \psi}{\partial \xi^{2}}-D \psi
$$

for the electrostatic potential $\phi^{(1)}=\psi$, where:

$$
\begin{aligned}
& A=\left(-\delta_{e}+\frac{3 V^{2}+\sigma_{1}}{\left(V^{2}-\sigma_{1}\right)^{3}}-\frac{\delta_{i} \mu^{2}}{\left(V^{2}-\sigma_{2}\right)^{2}}+\frac{4 V^{2} \delta_{i} \mu^{2}}{\left(V^{2}-\sigma_{2}\right)^{3}}\right) B, \\
& B=\frac{1}{2 V}\left(\frac{1}{\left(V^{2}-\sigma_{1}\right)^{2}}+\frac{\delta_{i} \mu}{\left(V^{2}-\sigma_{2}\right)^{2}}\right)^{-1}, \\
& C=V\left(\frac{\eta_{1}}{\left(V^{2}-\sigma_{1}\right)^{2}}+\frac{\delta_{i} \mu^{2} \eta_{2}}{\left(V^{2}-\sigma_{2}\right)^{2}}\right) B, \\
& D=V\left(\frac{\nu_{12}+\nu_{1 d}}{\left(V^{2}-\sigma_{1}\right)^{2}}+\frac{\delta_{i} \mu\left(\nu_{21}+\nu_{2 d}\right)}{\left(V^{2}-\sigma_{2}\right)^{2}}+\frac{\delta_{i} \nu_{21}+\mu \nu_{12}}{\left(V^{2}-\sigma_{1}\right)\left(V^{2}-\sigma_{2}\right)}\right) B .
\end{aligned}
$$

Recall that $\delta_{e}=1-\delta_{i}+s_{d} \delta_{d}$, while $V$ is given by Eq. (25).

The partial differential equation (PDE) (26) bears the form of a "hybrid" (mixed) Korteweg de Vries/Burgers equation (hKdV-B), with the addition of the extra (last) term in the $r h s$, in account of dissipation (damping). For $D=0$ (i.e., setting all of $\nu_{i j}$ to zero), this is a $\mathrm{KdV}$ Burgers equation, which yields an exact analytical solution in the form of a monotonic shock; see in Ref. [24] for details. Now, for $D \neq 0$, one may undertake a lengthy algebraic procedure, based on an ad hoc perturbative scheme [25], to find an approximate (damped shock) solution. The tedious algebraic procedure, omitted here for brevity, will be reported elsewhere in full detail [25]. The main relevant results are summarised in the following, to the extent that these are important in our analysis, to follow. Equation (26) in fact admits an (approximate) analytical solution in the form:

$$
\psi(\xi, \tau) \simeq \psi_{\max }(\tau)(1-Y)\left(1+\frac{1}{3} Y\right),
$$


with

$$
Y=\tanh \left(\frac{\xi-\xi_{0}-q(\tau) \tau}{L}\right),
$$

where $\xi_{0}$ is the initial position of the shock front and the shock solution's maximum amplitude is

$$
\psi_{\max }(\tau)=\frac{9 C^{2}}{25 A B} e^{-D \tau},
$$

its velocity is

$$
q(\tau)=\frac{9 C^{2}}{25 B D}\left(1-e^{-D \tau}-\frac{1}{3} D \tau\right) / \tau
$$

and its width is given by

$$
L=\frac{10 B}{C} e^{\frac{1}{6} D t} .
$$

This expression describes an electrostatic shock structure whose downstream asymptotic value exponentially decays in time as $\sim e^{-D \tau}$; see in Fig. 6. The entire structure slows down to a halt after a certain time, found after a numerical calculation to be $\simeq 2.821 D^{-1}$; the excitation will therefore be sufficiently long-lived, provided that $2.821 D^{-1} \gg \omega_{p}^{-1}$, or $D / \omega_{p} \ll 2.821$, i.e. practically speaking, for small values of the collisional coefficients $\nu_{i j}$ (note the definition of $D$ is given by Eqs. 27). Also, as we can notice, the width of the shock $L$, given by Eq. (32) as a function of time, becomes wider and wider due to the dissipative effect under the action of the damping coefficient $D$.

\section{PARAMETRIC ANALYSIS}

Based on the algebraic expressions Eq. 28 with Eqs. 27 , we have undertaken a detailed parametric investigation of the electrostatic shock's structural and dynamical features, in terms of various relevant plasma parameters.

\section{A. Phase velocity}

The effect of the negative ion density $\delta_{i}$ and the dust density $\delta_{d}$ on the phase velocity $V_{p h}$ of the nonlinear wave are shown in Figs. 3 and 4 . Recall that the multi-fluid plasma model adopted here supports two modes, namely fast and slow waves, as discussed in Figs. 3 and 4 . This is reflected in the (two) solutions of Eq. (25) for the phase speed. In Fig. 3, it is shown that the phase velocity of both modes increases, with an increase of the negative ion density. Fig. 4a shows that the phase velocity of the two nonlinear modes decreases with an increase in positive dust density. While an increase of the number density of negatively charged dust particles leads to an increase of the phase velocity.

\section{B. Electrostatic pulse polarity reversal}

The plasma density perturbation is related to the electrostatic potential perturbation through Eqs. (18)-(19).

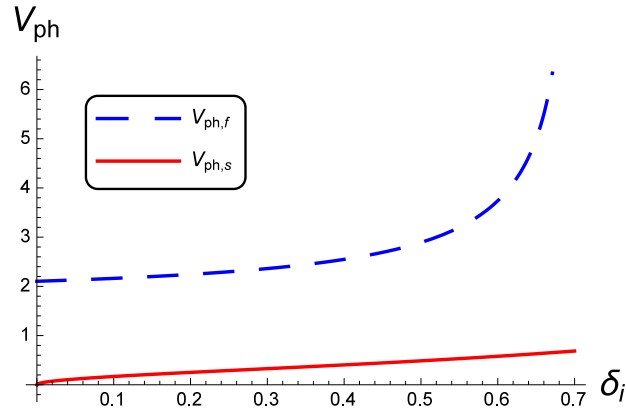

FIG. 3: (Color online) The effect of the negative ions (concentration) $n_{2,0}$ on the phase speed is investigated, taking as representative values: $s_{d}=-1, n_{d}=1.7 \times 10^{6} \mathrm{~cm}^{-3}$ and $n_{1,0}=2 \times 10^{9} \mathrm{~cm}^{-3}$. The other parameters are $z_{1}=z_{2}=1$, $z_{d}=350, m_{1}=39 m_{p}, m_{2}=146 m_{p}$ ( $m_{p}$ : proton mass), $T_{1}=8 T_{2}=T_{e}=0.2 \mathrm{eV}$. The dimensionless parameters are $\delta_{d}=0.3, \delta_{i}=0.5, \mu=0.267, \sigma_{1}=3$ and $\sigma_{2}=0.1$.

Eq. (18) shows that the sign of the density excitation, whether it represents a compression or a rarefaction in the positive-ion density perturbation, is associated with the sign (whether positive or negative, respectively) of the polarity of the electrostatic potential perturbation $\phi^{(1)}$. It is well known from previous works that the polarity of the electrostatic potential perturbation is determined by the sign of the coefficient of the nonlinear term $A$. In this article, this is reflected in Eq. (30) for $A$, which then enters (28). It is therefore clear that the sign of $A$ (positive or negative) will determine the polarity of the potential excitation. As shown in Fig. $5 \mathrm{a}$, for positively charged dust in the plasma (i.e., for $s_{d}=+1$ ), the value of $A$ is always positive, for any value of $\delta_{i}$. This means that only positive electrostatic potential pulses (and hence a compressive density disturbance) may exist. On the other hand, Fig. 5b shows that, for negatively charged dust particles, the value of the coefficient $A$ starts with a positive value and then changes sign to negative value at a critical value of the negative ion density, say $\delta_{i}=\delta_{i, c r}$.

In Figs. 6 and 7, we have depicted the full range of values for the parameters $\delta_{i}$ and $\delta_{d}$. The sign of the coefficient of the nonlinear term $(A)$ is depicted in Fig. 6 for the fast mode $\left(V=V_{+}\right)$, both for positive dust $\left(s_{d}=1\right.$, in Fig. 6a) and for negative dust charge $\left(s_{d}=\right.$ -1 , in Fig.6b). Likewise, the analogous plots for the slow mode $\left(V=V_{-}\right)$are provided in Fig. 7 . We note that polarity reversal is possible for the fast mode (only) (i.e. realized in the white regions in Fig. 6 and, in fact, nowhere in 7) is possible for both positive dust charge and for negative dust charge (primarily). We conclude that either compressive or rarefactive shock wave may exist, depending on whether the negative ions are a minority population or dominant. 


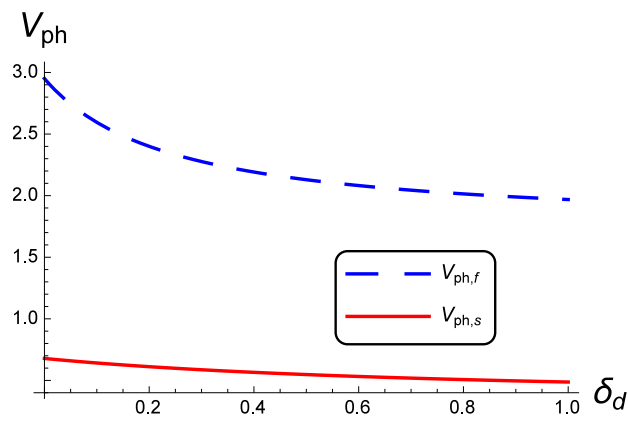

(a)

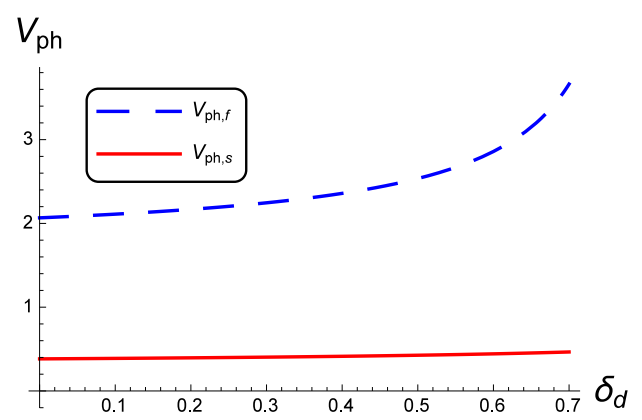

(b)

FIG. 4: (Color online) The phase velocity: (a) the effect of positive dust density $s_{d}=1$, and (b) the effect of negative dust density $s_{d}=-1$. The other parameters are $n_{2,0}=1.6 \times 10^{9} \mathrm{~cm}^{-3}$ and $n_{1,0}=2 \times 10^{9} \mathrm{~cm}^{-3}, z_{1}=z_{2}=$ $1, z_{d}=350, m_{1}=39 m_{p}, m_{2}=146 m_{p}$ ( $m_{p}$ : proton mass), $T_{e}=T_{1}=0.2 \mathrm{eV}, T_{2}=T_{e} / 8$. The dimensionless parameters are $\delta_{d}=0.3, \delta_{i}=0.5, \mu=0.267, \sigma_{1}=3$ and $\sigma_{2}=0.1$.

\section{Evolution of an electrostatic shock}

The temporal evolution of the electrostatic potential $\psi(\xi, \tau)$ and the associated electric field $E(\xi, \tau)$ is investigated in Figs. 8, 10 and 12. We have plotted the evolution of the electostatic potential (shock) structure for the fast mode in Fig. 10 and for the slow mode in Fig. 12. Based on the analytical solution Eq. (28), the electrostatic potential and the associated electric field are shown in Figs. 8, 10 and 12)(a, b) for different physical parameters. By using

$\psi(\xi, 0)=\frac{9 C^{2}}{25 A B}\left[1-\tanh \left(\xi-\xi_{0}\right)\right]\left[1+\frac{1}{3} \tanh \left(\xi-\xi_{0}\right)\right]$,

as initial condition, we have integrated Eq. (26) numerically by using a semispectral method [25]. The numerical result is shown in Figs. 8, 8 and 12)(c, d). The comparison between the analytical solution and the numerical outcome shows a very good agreement.We can also notice that the thickness of the shock wave increases as

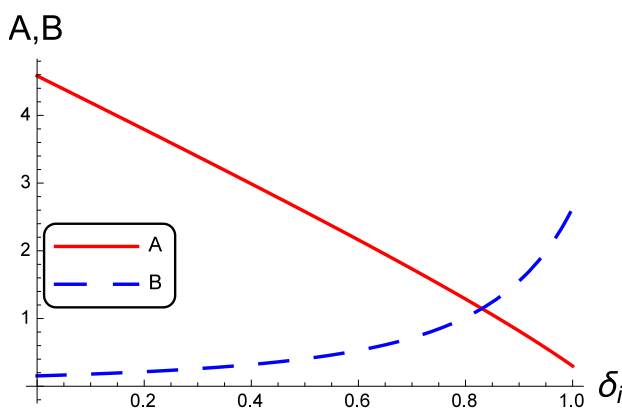

(a)

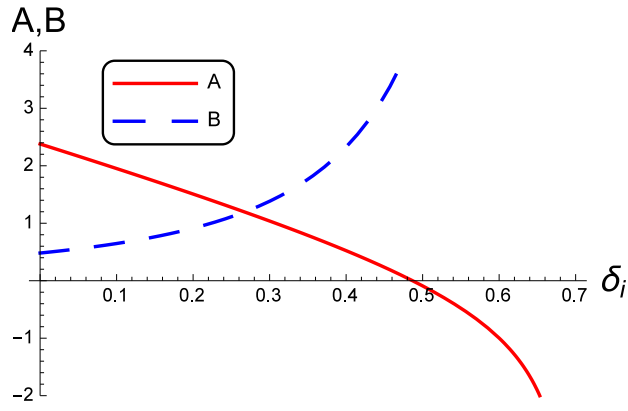

(b)

FIG. 5: (Color online) The coefficient of the nonlinear term ( $A$, continuous red curve) and the dispersion term $(B$, dashed blue curve) versus the ion density ratio $\delta_{i}$ : (a) the effect of positive dust density $s_{d}=1$, and (b) the effect of negative dust density $s_{d}=-1$. The other parameters are $n_{2,0}=1.6 \times 10^{9} \mathrm{~cm}^{-3}$ and $n_{1,0}=2 \times 10^{9} \mathrm{~cm}^{-3}, z_{1}=z_{2}=$ $1, z_{d}=350, m_{1}=39 m_{p}, m_{2}=146 m_{p}$ ( $m_{p}$ : proton mass), $T_{e}=T_{1}=0.2 \mathrm{eV}, T_{2}=T_{e} / 8$. The dimensionless parameters are $\delta_{d}=0.3, \delta_{i}=0.5, \mu=0.267, \sigma_{1}=3$ and $\sigma_{2}=0.1$.

time progresses, due to dissipation. The amplitude and the velocity of the wave are depicted in Figs. 9, 11 and 13, for three typical situations (parameter sets) considered. Clearly, both amplitude and velocity decrease for stronger damping, i.e. for higher values of the coefficient $D$.

\section{CONCLUSIONS}

We have introduced a hydrodynamical model, to model the dynamics of electrostatic shock waves in a plasma consisting of positive and negative ions, in addition to electrons and (either positively or negatively charged) dust particles. The linear dispersion relation was shown to admit a pair of ("fast" and "slow") acoustic modes. For finite amplitude perturbations, we have used a perturbation method to derive the governing equation for the electrostatic potential perturbation. Depending on 


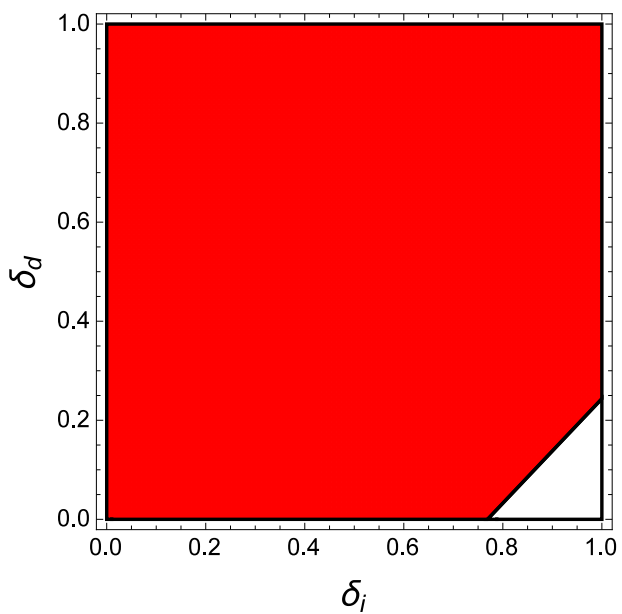

(a)

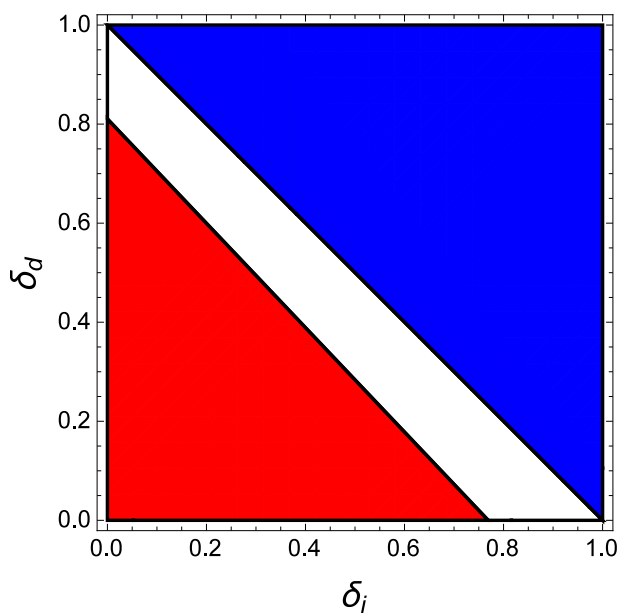

(b)

FIG. 6: (Color online) The sign of the nonlinearity coefficient $(A)$ is depicted, for the fast mode $V=V_{+}$, for (a) positive dust $\left(s_{d}=1\right)$, and for (b) negative dust $\left(s_{d}=-1\right)$, for $\eta_{1}=0.2, \eta_{2}=0.1, \nu_{12}=0.02, \nu_{1 d}=0.05, \nu_{2 d}=0.03, \sigma_{1}=3, \sigma_{2}=0.1$ and $\mu=0.267$. The shaded (red) region is for $A>0$, while the white region is for $A<0$ and the blue region (top right, in the right panel) for the forbidden region $\left(\delta_{e}=<0\right)$; cf. (12).

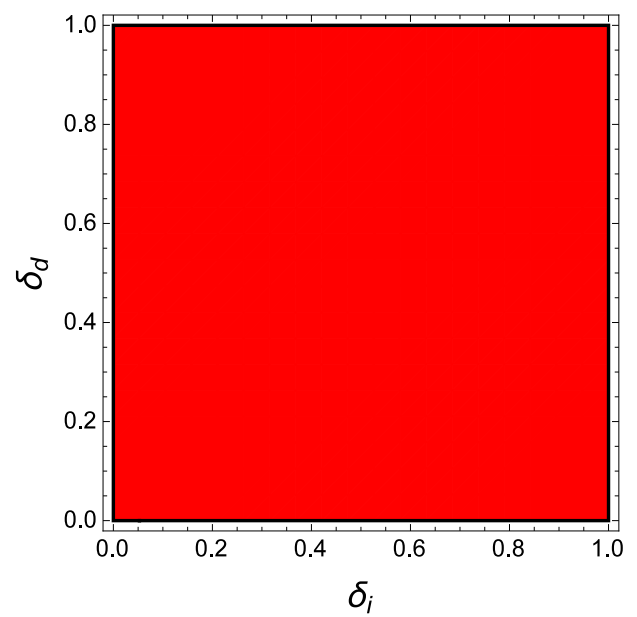

(a)

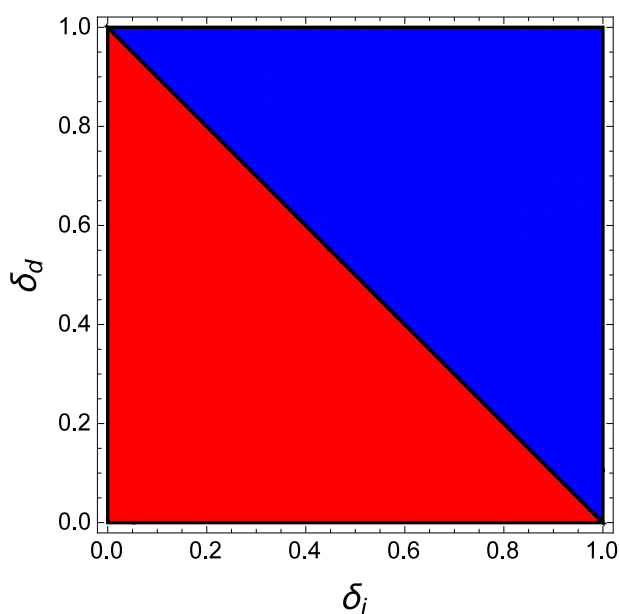

(b)

FIG. 7: (Color online) The sign of the nonlinearity coefficient $(A)$ is depicted, for the slow mode $V=V_{-}$, for (a) positive dust $\left(s_{d}=1\right)$, and for (b) negative dust $\left(s_{d}=-1\right)$, for $\eta_{1}=0.2, \eta_{2}=0.1, \nu_{12}=0.02, \nu_{1 d}=0.05, \nu_{2 d}=0.03, \sigma_{1}=3, \sigma_{2}=0.1$ and $\mu=0.267$. The shaded (red) region (entire left panel, bottom left part in the right panel) is for $A>0$, while the blue region (top right, in the right panel) is the forbidden region $\left(\delta_{e}=<0\right)$; cf. (12). We see that there is no negative $A$ region, essentially, to be associated with the slow mode.

the negative ion density, either compressive or rarefactive electrostatic potential excitations may exist. We have shown that the shock structure amplitude decreases exponentially, under the effect of dissipation, as intuitively expected.

Our results aim at casting some light to the dynamics of shocks in dissipative binary ion mixtures, in the pres- ence of dust. In particular, the role of the dust may be crucial in actually defining the shock polarity (here determined by the sign of the nonlinearity coefficient $A$, within our model). Likewise, injecting negative ions in a dusty plasma may affect the properties of electrostatic excitations which, in the presence of dissipation, may propagate in the form of electrostatic shocks. 


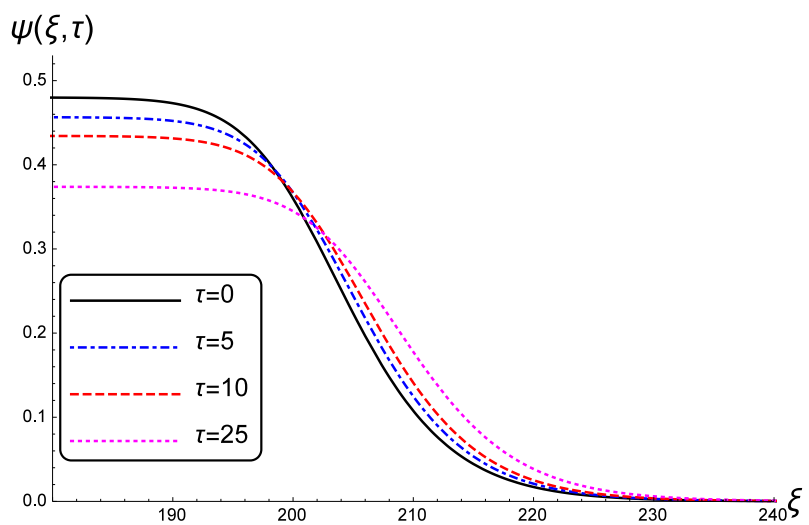

(a)

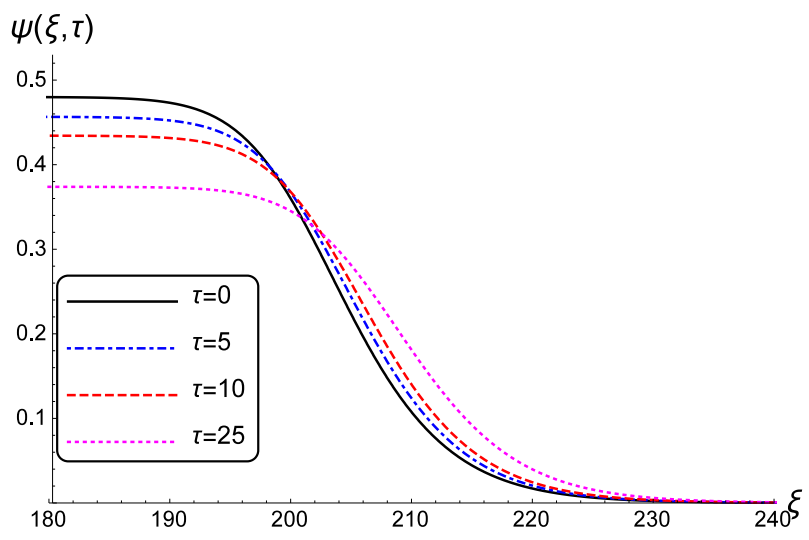

(c)

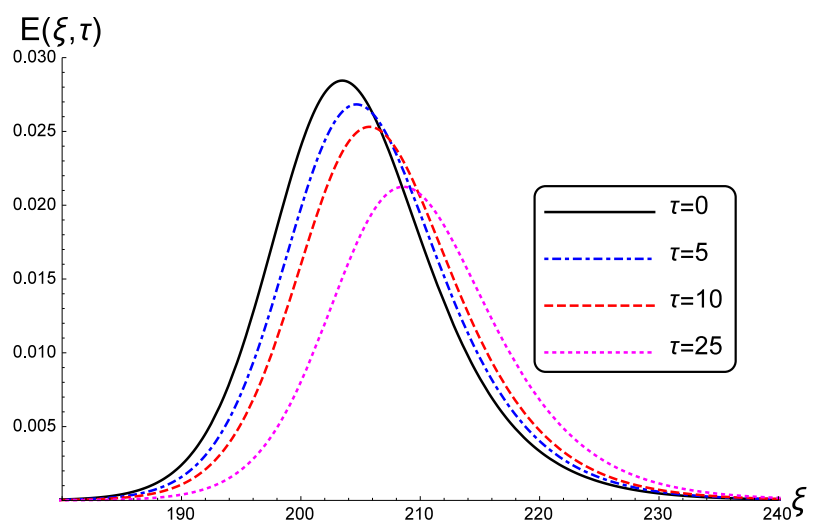

(b)

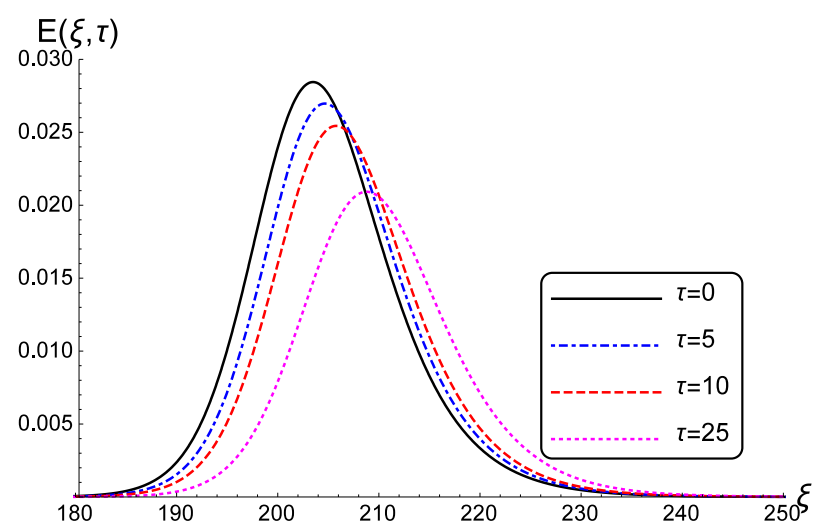

(d)

FIG. 8: (Color online) The temporal evolution of the electrostatic potential shock structure $\psi(\xi, \tau)$ is depicted, as it results from (a) the analytical model, based on the solution (28), and (c) the numerical simulation. The associated electric field $E(\xi, \tau)$ is respectively shown, i.e. as obtained from (b) the analytical and (d) the numerical result. An $a d$ hoc set of values have been taken for the coefficients as $A=1, B=1, C=1$ and $D=0.01$.

\section{Acknowledgments}

I.S.E. acknowledges financial support via an Egyptian Government fellowship. I.K. warmly acknowledges the hospitality provided by the Institute of Theoretical Physics (IFT) at the State University of São Paulo (UNESP), and is also grateful for support from the Foundation for Research Support of the State of São Paulo (FAPESP), in the form of a Visiting Researcher fellowship, during the latter stages of this work. A number of stimulating discussions with Professor Roberto Kraenkel (IFT, Brazil) and with Dr Brian Reville (Queen's University Belfast, UK) are gratefully acknowledged.

\section{APPENDIX A: DERIVATION OF KDVB EQUATION}

In order to derive KdVB equation (26), first we have introduced the coordinate transformation (16), from $\{x, t\}$ to $\{\xi, \tau\}$, onto Eqs. (6)-(10). Then, we have perturbed the plasma quantities $\left\{n_{j}, u_{j}, \phi\right\}$ around the equilibrium state $\{1,0,0\}$, as described by Eqs. 17. Substituting, we have proceeded by collecting different powers of $\epsilon$. From 


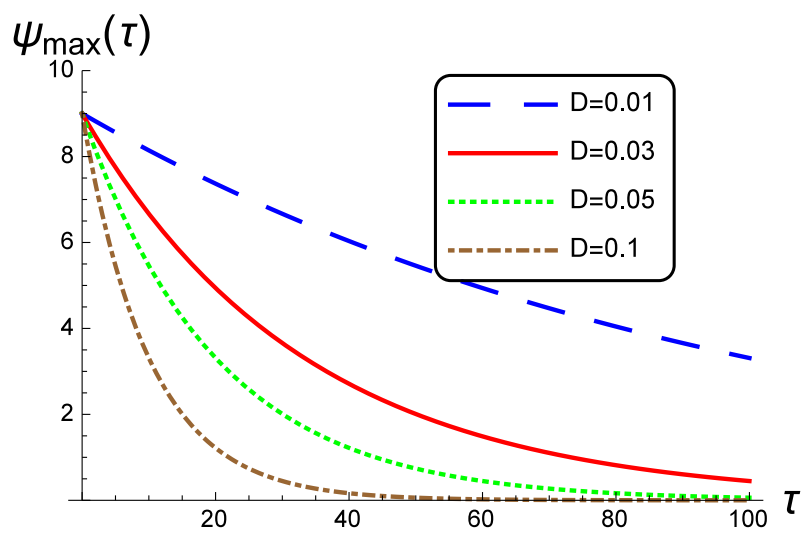

(a)

\section{$\mathrm{q}(\tau)$}

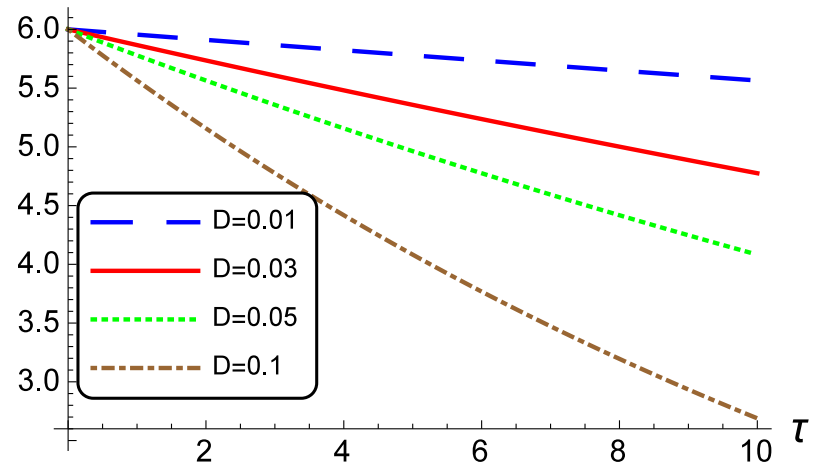

(b)

FIG. 9: (Color online) (a) The maximum amplitude and (b) the velocity of the electrostatic shock wave depicted in Fig.8. The coefficients are $A=1, B=1$ and $C=1$.

the lowest power in $\epsilon$, we obtain the following equations

$$
\begin{aligned}
-V \frac{\partial n_{1}^{(1)}}{\partial \xi}+\frac{\partial u_{1}^{(1)}}{\partial \xi} & =0 \\
-V \frac{\partial n_{2}^{(1)}}{\partial \xi}+\frac{\partial u_{2}^{(1)}}{\partial \xi} & =0 \\
-V \frac{\partial u_{1}^{(1)}}{\partial \xi}+\frac{\partial \phi^{(1)}}{\partial \xi}+\sigma_{1} \frac{\partial n_{1}^{(1)}}{\partial \xi} & =0 \\
-V \frac{\partial u_{2}^{(1)}}{\partial \xi}-\mu \frac{\partial \phi^{(1)}}{\partial \xi}+\sigma_{2} \frac{\partial n_{2}^{(1)}}{\partial \xi} & =0, \\
n_{1}^{(1)}-\delta_{i} n_{2}^{(1)}-\delta_{e} \phi^{(1)} & =0 .
\end{aligned}
$$

We have solved Eqs. (A1) - (A5), by using the boundary conditions $n_{1,2}^{(1)}=1$ and $u_{1,2}^{(1)}, \phi^{(1)} \rightarrow 0$ for $x \rightarrow \pm \infty$, to obtain the first order perturbed quantities $\left(n_{1,2}^{(1)}, u_{1,2}^{(1)}, \phi^{(1)}\right)$ in Eqs. (18) -(21).

The next highest (2nd-order) power in $\epsilon$ yields the following equations:

$$
\begin{aligned}
V \frac{\partial n_{1}^{(2)}}{\partial \xi}+\frac{\partial u_{1}^{(2)}}{\partial \xi} & =L_{1}, \\
V \frac{\partial n_{2}^{(2)}}{\partial \xi}+\frac{\partial u_{2}^{(2)}}{\partial \xi} & =L_{2}, \\
V \frac{\partial u_{1}^{(2)}}{\partial \xi}-\frac{\partial \phi^{(2)}}{\partial \xi}+\sigma_{1} \frac{\partial n_{1}^{(2)}}{\partial \xi} & =L_{3}, \\
V \frac{\partial u_{1}^{(2)}}{\partial \xi}+\mu \frac{\partial \phi^{(2)}}{\partial \xi}+\sigma_{2} \frac{\partial n_{1}^{(2)}}{\partial \xi} & =L_{4}, \\
n_{1}^{(2)}-\delta_{i} n_{2}^{(2)}-\delta_{e} \phi^{(2)} & =L_{5},
\end{aligned}
$$

where

$$
\begin{aligned}
L_{1} & =\frac{\partial n_{1}^{(1)}}{\partial \tau}+u_{1}^{(1)} \frac{\partial n_{1}^{(1)}}{\partial \xi}+n_{1}^{(1)} \frac{\partial u_{1}^{(1)}}{\partial \xi} \\
L_{2} & =\frac{\partial n_{2}^{(1)}}{\partial \tau}+u_{2}^{(1)} \frac{\partial n_{2}^{(1)}}{\partial \xi}+n_{2}^{(1)} \frac{\partial u_{2}^{(1)}}{\partial \xi} \\
L_{3} & =\frac{\partial u_{1}^{(1)}}{\partial \tau}+u_{1}^{(1)} \frac{\partial u_{1}^{(1)}}{\partial \xi}+\sigma_{1} n_{1}^{(1)} \frac{\partial n_{1}^{(1)}}{\partial \xi} \\
& -\nu_{12}\left(u_{1}^{(1)}-u_{2}^{(1)}\right)+\eta_{1} \frac{\partial^{2} u_{1}^{(1)}}{\partial x^{2}}-\nu_{1 d} u_{1}^{(1)} \\
L_{4} & =\frac{\partial u_{2}^{(1)}}{\partial \tau}+u_{2}^{(1)} \frac{\partial u_{2}^{(1)}}{\partial \xi}+\sigma_{2} n_{2}^{(1)} \frac{\partial n_{2}^{(1)}}{\partial \xi} \\
& -\nu_{21}\left(u_{2}^{(1)}-u_{1}^{(1)}\right)+\eta_{2} \frac{\partial^{2} u_{2}^{(1)}}{\partial x^{2}}-\nu_{2 d} u_{2}^{(1)} \\
L_{5} & =\frac{1}{2} \delta_{e} \phi^{(1) 2}-\frac{\partial^{2} \phi^{(1)}}{\partial \xi^{2}} .
\end{aligned}
$$

By substituting the first order quantities $\left(n_{1,2}^{(1)}, u_{1,2}^{(1)}\right)$ inside $L_{1}-L_{4}$ in Eqs. (A6) - (A9), we have solved Eq. (A6) and Eq. (A7) for $u_{1}^{(2)}$ and $u_{2}^{(2)}$. Then, by substituting $u_{1}^{(2)}$ and $u_{2}^{(2)}$ into Eqs. (A8) and (A9), we have solve them for $n_{1}^{(2)}$ and $n_{2}^{(2)}$ in terms of $\phi^{(1)}$. Finally, using Eq. (A10) and $n_{1,2}^{(2)}$, we have eventually obtained the KdV-Burgers equation (26). 


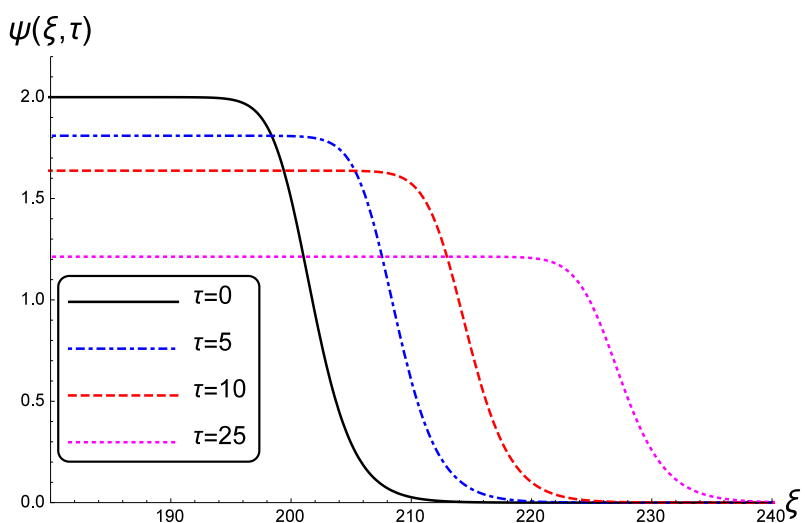

(a)

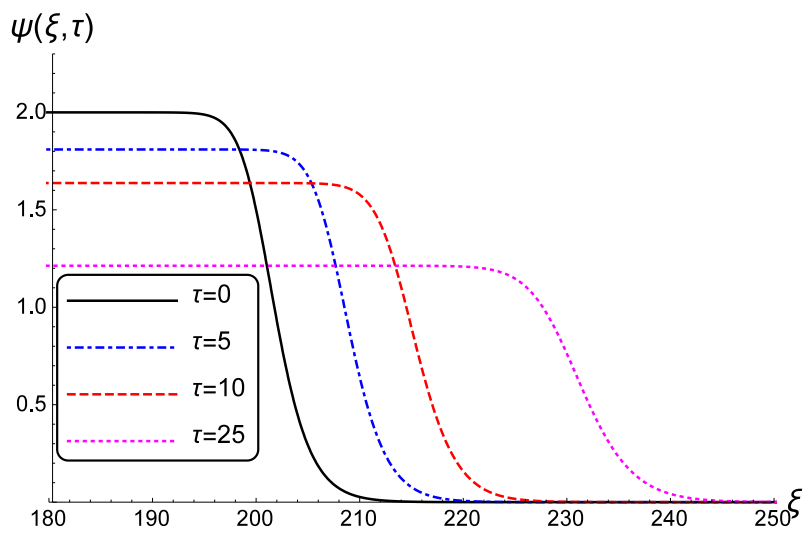

(c)

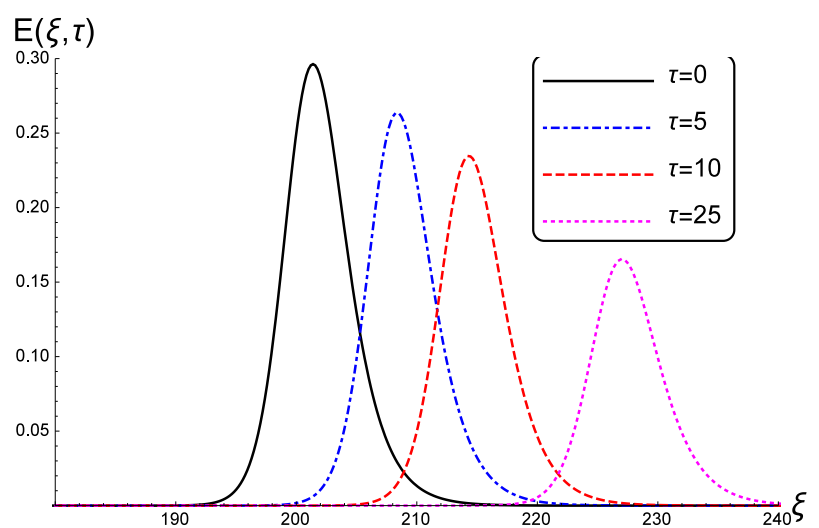

(b)

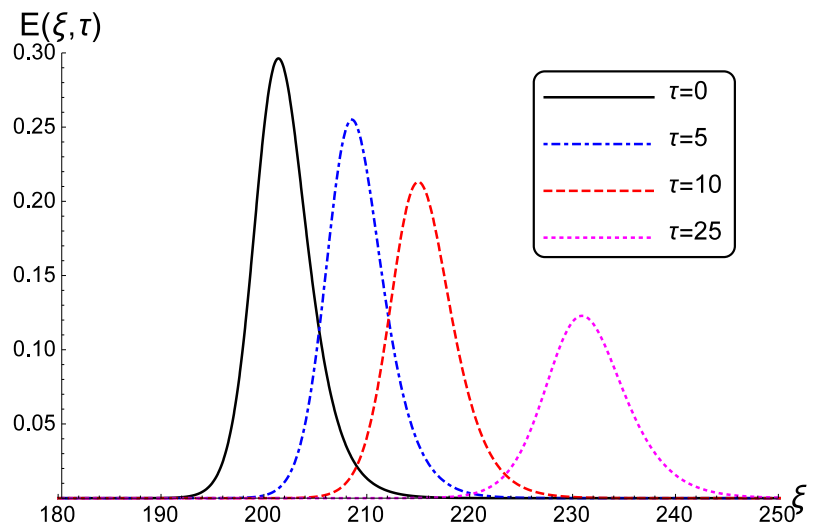

(d)

FIG. 10: (Color online) The temporal evolution of the electrostatic potential shock structure $\psi(\xi, \tau)$ is depicted, as it results from (a) the analytical model, based on the solution (28), and (c) the numerical simulation. The associated electric field $E(\xi, \tau)$ is respectively shown, i.e. as obtained from (b) the analytical and (d) the numerical result. Here, we have considered: $s_{d}=-1$ (negative dust), $\delta_{d}=0.3, \delta_{i}=0.5, \mu=0.267, \sigma_{1}=3, \sigma_{2}=0.1, \eta_{1}=5, \eta_{2}=4, \nu_{12}=0.02, \nu_{1 d}=0.05$ and $\nu_{2 d}=0.03$. The resulting coefficient values for the fast mode $\left(V_{+}=2.2\right)$ are: $A=1.5, B=1, C=2.5$ and $D=0.02$.

[2] Plasma Etching: An Introduction, edited by D.M. Manos and D.L. Flamm, Academic (New York, 1989).

[3] M.A. Lieberman and A.J. Lichtenberg, Principles of Plasma Discharges and Materials Processing, Wiley (New York, 1994).

[4] T. Takeuchi, S. Izuka, N. Sato, Phys. Rev. Lett. 80, 77 (1998).

[5] Q. Z. Luo, N. DAngelo, and R. L. Merlino, Phys. Plasmas 8, 2868 (1998).

[6] Y. Nakamura, H. Bailung, and P. K. Shukla, Phys. Rev. Lett. 83, 1602 (1999).

[7] P. Bandyopadhyay, G. Prasad, A. Sen, and P. K. Kaw, Phys. Rev. Lett. 101, 065006 (2008).

[8] A. Sarma, Y. Nakamura, Phy. Lett. A 373, 4174 (2009).

[9] J. Heinrich, S.-H. Kim, and R. L. Merlino, Phys. Rev. Lett. 103, 115002 (2009).

[10] S. K. Sharma, A. Boruah, Y. Nakamura, and H. Bailung, Phys. Plasmas 23, 053702 (2016).
[11] P. K. Shukla and A. A. Mamun, Introduction to Dusty Plasma Physics, Institute of Physics (Bristol, 2002).

[12] P. K. Shukla and A. A. Mamun, New J. Phys. 5, 17 (2003).

[13] V. E. Fortov and G. E. Morfill, Complex and Dusty Plasmas: From Laboratory to Space, CRC Press (New York, 2010).

[14] A. A. Mamun and P. K. Shukla, J. Plasma Phys. 77, 437 (2011).

[15] R. L. Merlino, J. Plasma Phys. 80, 773 (2014).

[16] P.K. Shukla, Phys. Plasmas 7, 1044 (2000).

[17] S. V. Vladimirov, K. Ostrikov, M. Y. Yu, and G. E. Morfill, Phys. Rev. E 67, 036406 (2003).

[18] M. Rosenberg, R.L. Merlino, Planet. Space Sci., 55, 1464 (2007).

[19] A. P. Misra, N. C. Adhikary, and P. K. Shukla, Phys. Rev. E 86, 056406 (2012).

[20] Y Nakamura, T Odagiri and I Tsukabayashi, Plasma 


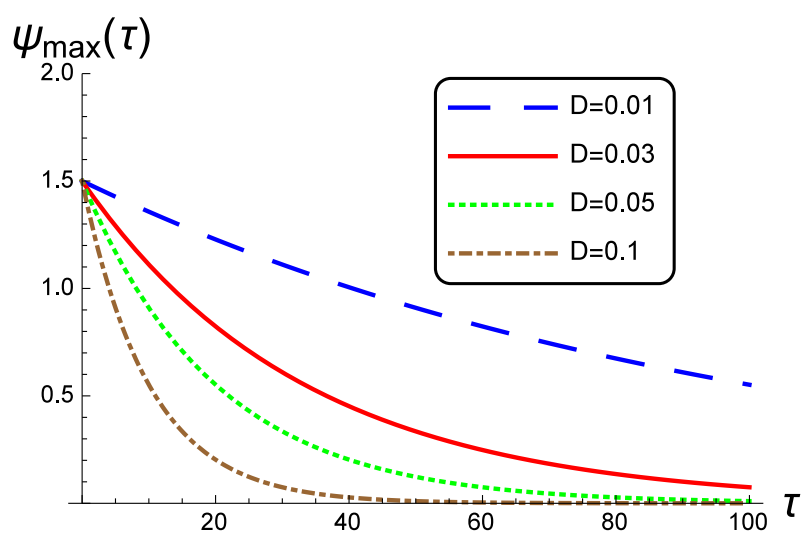

(a)

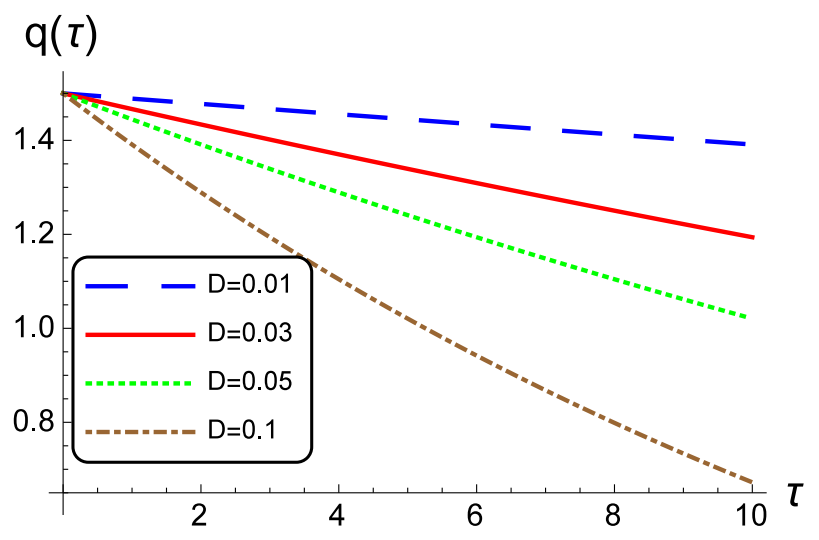

(b)

FIG. 11: (Color online) (a) The maximum amplitude and (b) the velocity of the electrostatic shock wave for structure shown in depicted in Fig.10, i.e., $s_{d}=1, \delta_{d}=0.3, \delta_{i}=0.5, \mu=0.267, \sigma_{1}=3, \sigma_{2}=0.1, \eta_{1}=5, \eta_{2}=4, \nu_{12}=0.02, \nu_{1 d}=0.05$ and $\nu_{2 d}=0.03$. The corresponding coefficient values for the fast mode $\left(V_{+}=2.2\right)$ are: $A=1.5, B=1$ and $C=2.5$.

Phys. Cont. Fus., 39, 105 (1997).

[21] R. Ichiki and M. Shindo, Phys. Plasmas 8, 4275 (2001).

[22] S. H. Kim, R.L. Merlino, Phys. Plasmas 13, 052118 (2006).

[23] H. Washimi, and T. Taniuti, Phys. Rev. Lett. 17, 996 (1966).

[24] I. Kourakis, S. Sultana and F. Verheest, Astrophys. Space
Sci. 338, 245 (2012).

[25] Generalized hybrid Korteweg de Vries - Burgers type equation for propagating shock structures in nonintegrable systems, by I. S. Elkamash, F. Verheest, R.A. Kraenkel, R.M. Coutinho, B. Reville and I. Kourakis, submitted to Nonlinear Dynamics, under review (2018). 


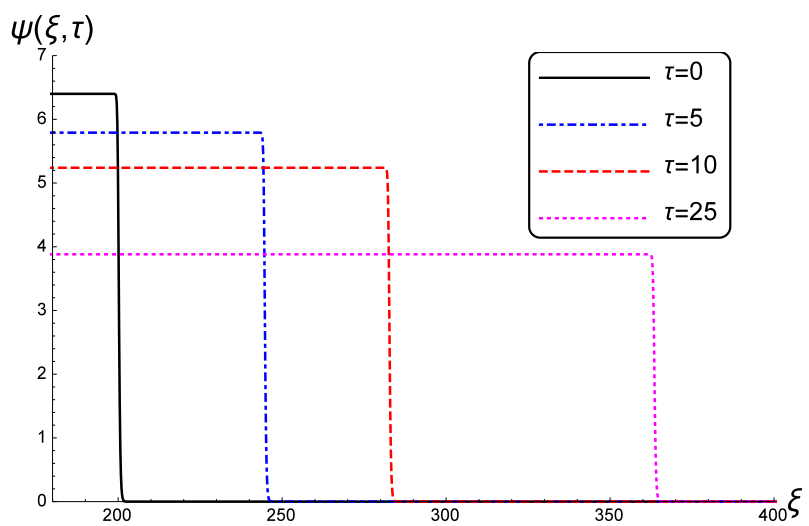

(a)

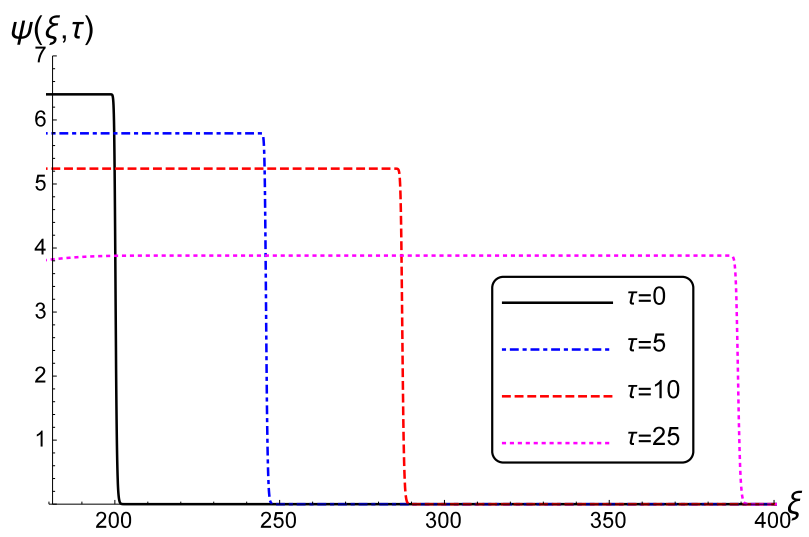

(c)

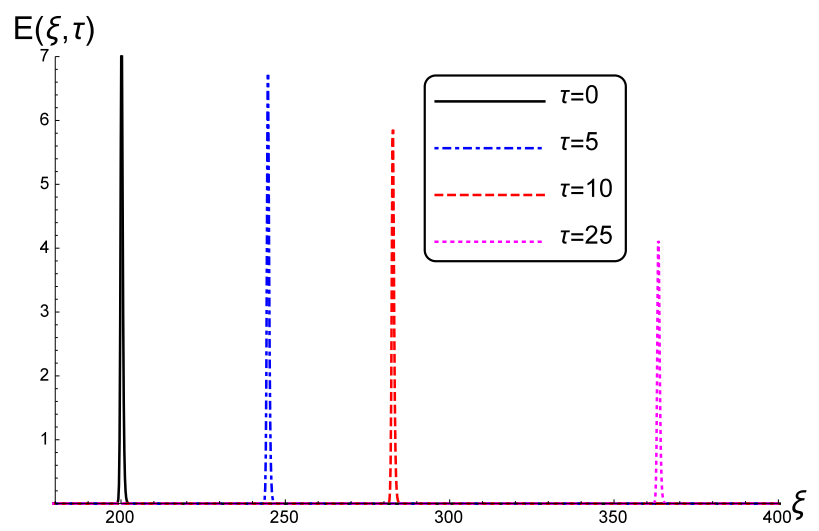

(b)

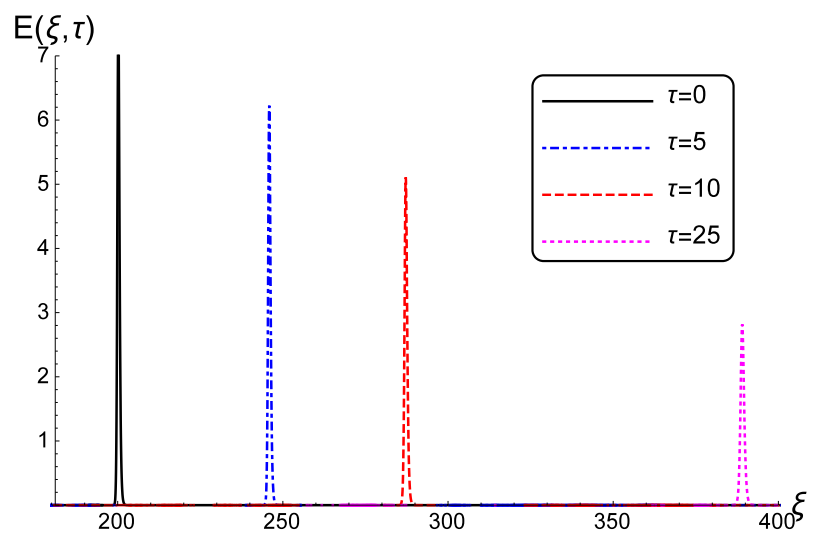

(d)

FIG. 12: (Color online) The temporal evolution of the electrostatic potential shock structure $\psi(\xi, \tau)$ is depicted, as it results from (a) the analytical model, based on the solution (28), and (c) the numerical simulation. The associated electric field $E(\xi, \tau)$ is respectively shown, i.e. as obtained from (b) the analytical and (d) the numerical result. Here, we have considered: $s_{d}=-1$ (negative dust), $\delta_{d}=0.3, \delta_{i}=0.5, \mu=0.267, \sigma_{1}=3, \sigma_{2}=0.1, \eta_{1}=5, \eta_{2}=4, \nu_{12}=0.02, \nu_{1 d}=0.05$ and $\nu_{2 d}=0.03$. The resulting coefficient values for the slow mode $\left(V_{-}=0.4\right)$ are: $A=3, B=0.1, C=2$ and $D=0.02$. 


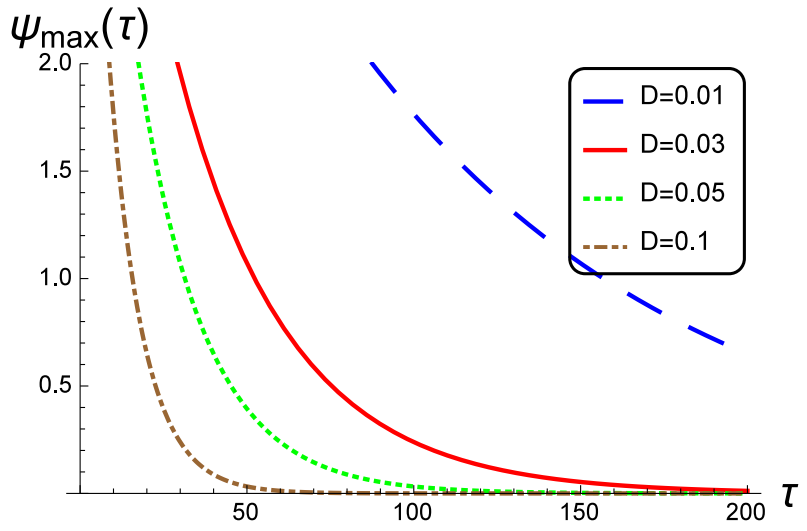

(a)

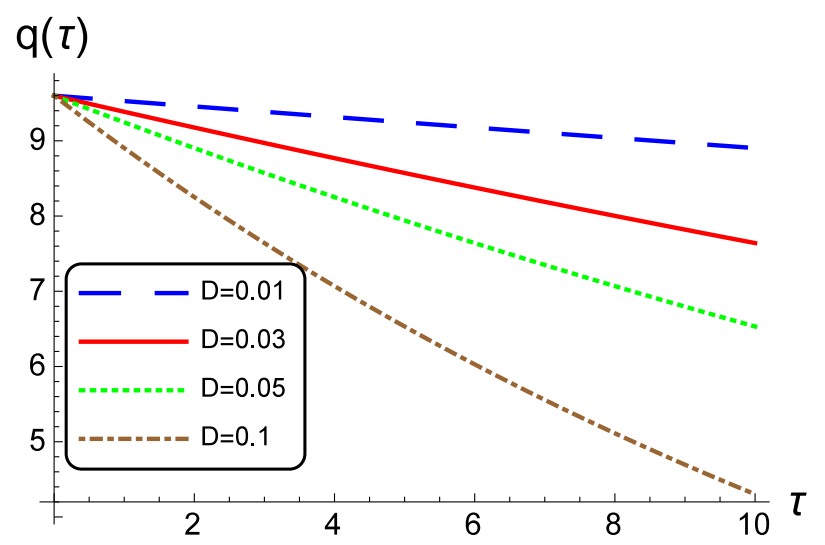

(b)

FIG. 13: (Color online) (a) The maximum amplitude and (b) the velocity of the electrostatic shock wave for structure shown in depicted in Fig.12, i.e., $s_{d}=1, \delta_{d}=0.3, \delta_{i}=0.5, \mu=0.267, \sigma_{1}=3, \sigma_{2}=0.1, \eta_{1}=5, \eta_{2}=4, \nu_{12}=0.02, \nu_{1 d}=0.05$ and $\nu_{2 d}=0.03$. The corresponding coefficient values for the slow mode $\left(V_{-}=0.4\right)$ are: $A=3, B=0.1$ and $C=2$. 